\title{
Das feline tracheale Lymphom
}

\author{
Nina Müller, Gabriel Wurtinger, Christina Schwens, Andreas Moritz
}

Das maligne Lymphom ist der häufigste hämatopoetische Tumor der Katze. Über die Therapie und Überlebenszeit des sehr selten vorkommenden trachealen Lymphoms ist jedoch nur wenig bekannt. In diesem Beitrag geben die Autoren eine Literaturübersicht und berichten über eigene Erfahrungen.

\section{Das feline Lymphom}

Bei der Katze sind circa 33\% aller Tumoren hämatopoetischer Natur und davon sind 90\% maligne Lymphome. Jährlich erkranken etwa 200 von 100000 Katzen [7]. Das feline Lymphom lässt sich nach unterschiedlichen Gesichtspunkten einteilen.

Aus anatomischer Sicht unterscheidet man:

- mediastinales Lymphom

- gastrointestinales Lymphom

- multizentrisches Lymphom

- extranodale Lymphome
$\mathrm{Zu}$ den extranodalen Formen zählen nasale, renale, zentrale, okuläre, dermale, laryngeale, pharyngeale und tracheale Lymphome [7].

Eine andere Einteilungsmöglichkeit kann histologisch oder zytologisch mittels Nachweis von CD3- oder CD79a-Antigen nach dem Immunophänotyp erfolgen. Dabei unterscheidet man das B-ZellLymphom und das T-Zell-Lymphom. Ferner kann ebenfalls histologisch oder zytologisch zwischen kleinzelligen und großzelligen sowie gut bis schlecht differenzierten Formen unterschieden werden.

\section{Das tracheale Lymphom - Literaturübersicht}

\section{Signalement und Anamnese}

Soweit den Autoren bekannt ist, sind bisher 9 Fallberichte eines trachealen Lymphoms veröffentlicht worden, in denen sich detaillierte Angaben zur Therapie und
Prognose finden $(\triangleright$ Tab. 1$)[1,2,3,4,6,9]$. Die Hauptsymptomatik der Tiere bestand anamnestisch in einer Dyspnoe. Weitere Symptome waren:

- Stridores

- Husten

- Tachypnoe

- Leistungsintoleranz

Das mediane Alter der Tiere lag bei 10,5 Jahren (4-13 Jahre) [1,2,3,6,9]. Die Tiere waren männlich $(\mathrm{n}=2)$, männlich-kastriert $(n=5)$ und weiblich-kastriert $(n=1)$. Unter den Katzen befanden sich 5 Europäisch Kurzhaar, 2 Siamkatzen und 1 Europäisch Langhaar. In einem Fall wurden keine Informationen zum Signalement genannt.

\section{Diagnostik}

In der klinischen Untersuchung fielen die Katzen hauptsächlich mit Dyspnoe sowie einem auskultatorischen Giemen auf. Einige waren zyanotisch.

Tab. 1 Felines tracheales Lymphom: Fallberichte aus der Literatur.

\begin{tabular}{|c|c|c|}
\hline Signalement & Therapie & Outcome \\
\hline Siamkatze, 7 J, männlich [6] & $\begin{array}{l}\text { Chirurgie } \\
\text { Chemotherapie (D, C, P) }\end{array}$ & 8 Monate, komplette Remission \\
\hline EKH, 11 J, männlich-kastriert [1] & Chirurgie & 4 Monate \\
\hline Siamkatze, 9 ], männlich [9] & Chirurgie & 10 Tage \\
\hline ELH, 10 J, weiblich-kastriert [2] & Glukokortikoide & 38 Tage \\
\hline EKH, 4 J, männlich-kastriert [2] & Chemotherapie (D, C, V, P) & 19 Monate, komplette Remission \\
\hline EKH, 11 J, männlich-kastriert [2] & $\begin{array}{l}\text { Chemotherapie (P, V } 1 \times \text { ) } \\
\text { später Bestrahlung }\end{array}$ & 17 Monate, komplette Remission \\
\hline EKH, 13 J, männlich-kastriert [2] & $\begin{array}{l}\text { Chemotherapie (C, V, P) } \\
\text { Bestrahlung }\end{array}$ & 8 Monate, komplette Remission (Hautlymphom) \\
\hline keine Angaben [4] & $\begin{array}{l}\text { Tracheostomie } \\
\text { Bestrahlung } \\
\text { Chemotherapie (C, V, P, L-Asparaginase) }\end{array}$ & 134 Tage (80-183 Tage) \\
\hline EKH, 13 J, männlich-kastriert [3] & Chemotherapie (D, C, V, P) & 15 Monate, komplette Remission \\
\hline
\end{tabular}


Zur weiteren Diagnostik wurden bei allen Katzen Röntgenaufnahmen des Thorax angefertigt. Bei 3 Tieren wurde in der Röntgenaufnahme eine weichteildichte Einengung der Trachea über einen längeren Abschnitt gesehen [2,3]. Bei 2 weiteren Katzen konnte röntgenologisch eine solide Masse in der Trachea gesehen werden und eine weitere Katze hatte zusätzlich eine Masse im rechten kaudalen Thorax [2]. Bei den übrigen 3 Katzen wurde eine tracheale Masse vermutet $[1,8,11]$. Dugas et al. fertigten zusätzlich noch eine CT an [3]. Hierbei wurde eine $8 \mathrm{~cm}$ lange Masse, die die Trachea von dorsal einengte, dargestellt [3]. In den meisten Fällen (5/9) wurde eine Tracheoskopie durchgeführt.

Die endgültige Diagnose wurde mittels zytologischer (9/9) und histologischer Untersuchungen (3/9) gestellt. In 3 von 9 Fällen erfolgte eine Bürstenzytologie, bei einem Patienten eine bronchoalveoläre Lavage. Im Falle der soliden Masse wurde eine Feinnadelaspiration mit zytologischer Untersuchung durchgeführt. In 3 von 9 Fällen wurden zusätzlich Biopsien histopathologisch untersucht, die die zytologische Diagnose bestätigten [2]. Da die Entstehung von Lymphomen im Zusammenhang mit einer FeLV- oder FIVInfektion stehen kann, wird die Untersuchung dieser Erkrankungen empfohlen. Von 8 getesteten Tieren war eine Katze FeLV-positiv [6], die restlichen Tiere waren sowohl FeLV- als auch FIV-negativ.

\section{Therapie und Prognose}

Für das tracheale Lymphom gibt es aufgrund des seltenen Vorkommens nur wenige Angaben über die Therapieoptionen und die damit verbundenen Überlebenszeiten.

Allgemein wird das Lymphom der Katze mittels einer Chemotherapie behandelt, häufig ist die Kombination verschiedener Chemotherapeutika indiziert. Die Ansprechrate liegt jedoch lediglich bei $50-80 \%$ und es wird eine mediane Überlebenszeit von 4-6 Monaten genannt [8]. Wichtig ist aber trotzdem eine Unterscheidung der verschiedenen Formen, da dies einen wesentlichen Einfluss auf das Outcome und die anschließende Therapie haben kann.
Bei nasalen Lymphomen kann sowohl eine Chemotherapie als auch eine Bestrahlungstherapie indiziert sein. Insgesamt ist für diese Tiere eine mediane Überlebenszeit von 1,5-3 Jahren beschrieben. Im Gegensatz dazu haben zentrale Lymphome die schlechteste Prognose mit einer medianen Überlebenszeit von 1-2 Monaten trotz einer Kombinationschemotherapie.

Bei der gastrointestinalen Form ist eine histologische Unterscheidung sinnvoll. Es wird zwischen klein- und großzelligen Lymphomen sowie zwischen einer transmuralen oder muralen Verteilung der entarteten Zellen unterschieden. Es konnte gezeigt werden, dass kleinzellige, murale T-Zell-Lymphome im Gastrointestinaltrakt eine gute Prognose mit wenig aggressiven Chemotherapieprotokollen haben [8].

Die Unterscheidung der verschiedenen

Lokalisationen ist von wichtiger

Bedeutung für die Therapie und

Prognose der Lymphome.

Beim trachealen Lymphom wurden verschiedene Therapien mit unterschiedlichem Erfolg eingesetzt. So wurde z. B. bei 2 von 9 Katzen eine chirurgische Therapie gewählt. Die mittlere Überlebenszeit lag bei 65 Tagen $[1,9]$. Eine Kombination aus chirurgischer Therapie und systemischer Chemotherapie unter der Verwendung von Doxorubicin, Cyclophosphamid und Prednisolon erzielte eine komplette Remission des Tumors über 8 Monate [6]. Die alleinige Gabe von Glukokortikoiden führte zu einer Überlebenszeit von 38 Tagen [2]. Jakubiak et al. konnten bei 4 Tieren mit laryngealen oder trachealen Massen bei Kombination verschiedener Therapieansätze eine mediane Überlebenszeit von 134,5 Tagen erzielen [4]. Die Katze mit dem trachealen Lymphom bekam eine Tracheostomie sowie eine Kombination aus Bestrahlung und Chemotherapie. Des Weiteren wurde bei 2 Tieren zunächst mit einer Kombinationschemotherapie begonnen, die aufgrund der Nebenwirkungen abgebrochen werden musste. Die Besitzer entschieden sich dann für eine Bestrahlungstherapie. Eine dieser Katzen zeigte hiermit eine komplette Remission von 17 Monaten. 

pnoe, entwickelte unter der Therapie aber ein kutanes Lymphom [2]. Eine Kombinationschemotherapie wurde bei weiteren 2 Katzen durchgeführt. Es konnte eine mittlere Überlebenszeit von 17 Monaten erreicht werden [2,3].

\section{Diskussion}

\section{Signalement und Anamnese}

Dyspnoe war bei allen Katzen der hauptsächliche Grund für die Vorstellung in der Praxis. Das mediane Alter der Tiere lag bei 10,5 Jahren. Männliche Tiere waren überrepräsentiert (7/9), wobei die meisten kastriert waren (5/7). Mediastinale Lymphome sind bei Siamkatzen im Allgemeinen überrepräsentiert, wobei die Diagnose häufig in jungen Jahren gestellt wird [8]. Europäisch Kurzhaar sind den Fallberichten zufolge am häufigsten betroffen (5/9).

\section{.konkret}

Männliche Katzen sind mit einem Verhältnis von 1,5:1 häufiger von einem Lymphom betroffen als weibliche [8].

\section{Diagnostik}

In der klinischen Untersuchung zeigten alle Katzen Dyspnoe. Des Weiteren war auskultatorisches Giemen sehr häufig (4/9). Die Inzidenz der FeLV-positiven Katzen sinkt aufgrund der Impfungen deutlich, so auch die der FeLV-assoziierten Lymphome [8]. Unter den bisher beschriebenen Fallberichten des trachealen
Die andere zeigte 8 Monate keine Dys-

Lymphoms war lediglich eine Katze FeLV-positiv [6].

Röntgenologisch konnte bei den Katzen selten eine solide Masse in der Trachea festgestellt werden. Meistens gaben die Röntgenaufnahmen Hinweise auf eine weichteildichte Einengung der Trachea über unterschiedlich große Abschnitte [2].

Die Diagnosestellung eines felinen Lymphoms erfolgte mithilfe einer zytologischen Untersuchung (9/9) der Feinnadelaspiraten oder mithilfe einer histologischen Untersuchung von Biopsien (3/9) der veränderten Organe oder Lymphknoten. In der Literatur wird immer wieder von Diskrepanzen zwischen der zytologischen und histologischen Diagnose berichtet. Für Rundzelltumoren ist die Korrelation in der Regel sehr gut, aber abhängig vom untersuchten Organ. Braun et al. konnten bei $63,7 \%$ der zytologisch untersuchten Lymphknoten eine Übereinstimmung finden, wobei hier nicht nur Rundzelltumoren eingeschlossen waren. Im Vergleich war die Korrelation bei Milz und Haut deutlich schlechter [5].

Bisweilen werden zur Differenzierung des Lymphoms der Katze weitere diagnostische Tests wie die Immunhistologie, die Durchflusszytometrie oder die Klonalitätsbestimmung eingesetzt [8]. Jedoch ist dies zurzeit noch eher von wissenschaftlichem Interesse für das tracheale Lymphom, da eine solche Untersuchung - im Gegensatz zum Hund

Tab. 2 Ergebnisse der Blutuntersuchung.

\begin{tabular}{|l|l|}
\hline Parameter & gemessener Wert (Referenzwert) \\
\hline Leukozyten & $5,79 \mathrm{G} / \mathrm{l}(6-18 \mathrm{G} / \mathrm{l})$ \\
\hline Lymphozyten & $0,44 \mathrm{G} / \mathrm{l}(1,5-7 \mathrm{G} / \mathrm{l})$ \\
\hline Retikulozyten & $45,1 \mathrm{G} / \mathrm{I}(<40 \mathrm{G} / \mathrm{l})$ \\
\hline Glukose & $8,14 \mathrm{mmol} / \mathrm{I}(3,89-6,11 \mathrm{mmol} / \mathrm{l})$ \\
\hline Cholesterin & $3,99 \mathrm{mmol} / \mathrm{I}(2,46-3,37 \mathrm{mmol} / \mathrm{l})$ \\
\hline Triglyceride & $2,26 \mathrm{mmol} / \mathrm{I}(0,57-1,14 \mathrm{mmol} / \mathrm{l})$ \\
\hline alkalische Phosphatase & $86 \mathrm{U} / \mathrm{I}(<39,7 \mathrm{U} / \mathrm{I})$ \\
\hline GLDH & $13 \mathrm{U} / \mathrm{I}(<11,3 \mathrm{U} / \mathrm{l})$ \\
\hline ALT & $225 \mathrm{U} / \mathrm{I}(<70 \mathrm{U} / \mathrm{l})$ \\
\hline
\end{tabular}

- nicht über die weitere Therapie entscheidet.

Allgemein gilt bei einem Lymphom die Chemotherapie als Mittel der Wahl. Lokalisierte Lymphome wie das nasale Lymphom zeigen ein besseres Ansprechen mit einer Bestrahlungstherapie [8]. Über das tracheale Lymphom ist diesbezüglich nur wenig bekannt.

Die Therapie mittels Bestrahlung oder Chemotherapeutika erbrachte im

Vergleich zur chirurgischen Therapie oder alleinigen Gabe von Glukokortikoiden lange Überlebenszeiten [2,3].

Die Chemotherapie bietet dabei den Vorteil, dass sie systemisch wirksam ist und man bei einem Lymphom eine systemische Ausbreitung nie sicher ausschließen kann. Die Bestrahlung wirkt hingegen nur lokal. Zum Beispiel entwickelte eine Katze unter der Bestrahlungstherapie ein kutanes Lymphom [2]. Die Verträglichkeit einer Chemotherapie bei der Katze wird in der Regel als gut beschrieben [8]. Kommt es jedoch zu starken Nebenwirkungen, stellt die Bestrahlungstherapie sicherlich eine Alternative dar.

Die Überlebenszeiten mit einer chirurgischen Therapie oder einer alleinigen Gabe von Glukokortikoiden waren sehr kurz $[1,2,9]$. Eine chirurgische Entfernung eines trachealen Tumors stellt einen sehr invasiven Eingriff dar. In einer Fallserie von 27 Katzen mit laryngealen, laryngotrachealen oder trachealen Massen überlebten die Katzen mit einer Tracheostomie median 3 Tage [4].

\section{Fallbericht}

\section{Signalement und Anamnese}

Eine 9 Jahre alte, weiblich-kastrierte Europäisch Kurzhaar Katze wurde in der Klinik vorgestellt. Sie wurde als reine Wohnungskatze gehalten und wies einen unregelmäßigen Impf- und Entwurmungsstatus auf. Zwei Monate zuvor wurde sie aufgrund betont abdominaler Atmung, zeitweise auftretenden $\mathrm{He}$ chelns ohne Zyanose sowie Leistungsschwäche beim Haustierarzt vorgestellt. Der Patient wurde 2-mal im Abstand von 14 Tagen mit Methylprednisolon (Depo- 


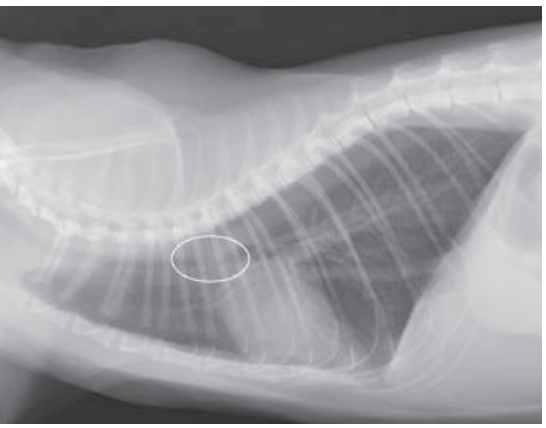

Abb. 1 Laterolaterale Röntgenaufnahme des Thorax zum Zeitpunkt der Erstvorstellung: Kraniodorsal der Herzbasis befindet sich eine $1 \times 0,5 \mathrm{~cm}$ große weichteildichte Verschattung, die die Trachea fast in ihrem ganzen Durchmesser überlagert. Das Lungenfeld ist erweitert.

(C) Klinik für Kleintiere, Innere Medizin, JLU Gießen

Medrate $\left.^{\circledR}\right)$ behandelt, was zu einer Verbesserung der Symptome führte. Jedoch kam es je 2 Wochen nach der Injektion $\mathrm{zu}$ einem Rezidiv. Am Tag vor der Vorstellung in der Klinik wurde die Katze erneut mit Dyspnoe bei einem weiteren Tierarzt vorgestellt.

\section{Diagnostik}

\section{Allgemeine Untersuchung}

Die Katze wies bei der Vorstellung ein mittelgradig gestörtes Allgemeinbefinden auf und war geringgradig adipös. Sie zeigte eine gemischte Dyspnoe mit einer Atemfrequenz von 50 Atemzügen/Minute und einen abdominalen Atemtyp. Die Körperinnentemperatur betrug $38,6^{\circ} \mathrm{C}$. Die Schleimhäute waren blassrosa und die kapilläre Rückfüllzeit lag unter 2 Sekunden. Die Palpation der peripheren Lymphknoten war ohne besonderen Befund. Im Abdomen wurde ein aufgegaster Magen ertastet. Bei der Auskultation konnte eine regelmäßige Herzfrequenz von 200 Schlägen/Minute ohne Nebengeräusche festgestellt werden. Die Lunge war in- und exspiratorisch verschärft.

\section{Weiterführende Diagnostik}

Röntgenologisch fand sich in der laterolateralen Aufnahme des Thorax eine $1 \times 0,5 \mathrm{~cm}$ große weichteildichte rundliche Masse im Lumen der Trachea, die im 5.- 6. Interkostalraum lokalisiert war. Des Weiteren wurden eine mittelgradige bronchiale Lungenzeichnung sowie ein erweitertes Lungenfeld diagnostiziert $($ Abb. 1).

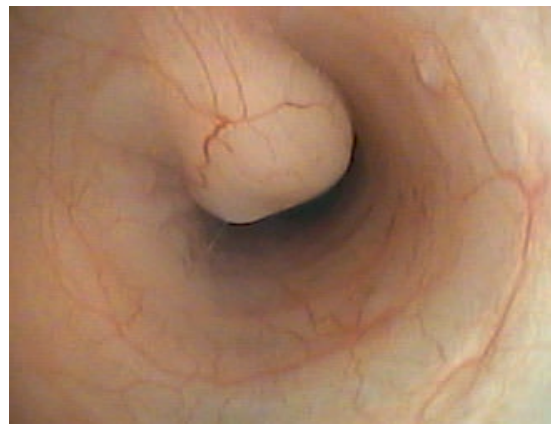

Abb. 2 Tracheoskopie: intraluminale Masse, die von dorsal hineinragt. Sie ist von gleicher Oberflächenstruktur wie die umliegende Schleimhaut.

(c) Klinik für Kleintiere, Innere Medizin, JLU Gießen

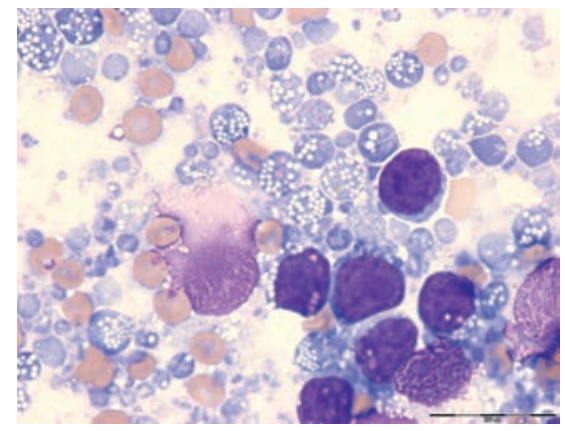

Abb. 3 Abklatschzytologie der intratrachealen Masse. Es finden sich einige bis zahlreiche kleine bis mittelgroße monomorphe lymphatische Blasten mit rundovalem Nukleolus mit feinklumpiger Chromatinstruktur, sichtbaren Makronukleoli und zum Teil hellen, scharf umschriebenen intrazytoplasmatischen Vakuolen. Zusätzlich sind zahlreiche mittelgroße bis große Zytoplasmaabschnürungen lymphatischer Zellen zu sehen, die zumeist zahlreiche helle Vakuolen enthalten (Ölimmersion, $1000 \mathrm{x}$, May-Grünwald-Giemsa-Färbung). (c) Zentrallabor Klinikum Veterinärmedizin, JLU Gießen 
In den Blutuntersuchungen konnten folgende Abweichungen festgestellt werden $(\triangleright$ Tab. 2):

- ggr. Leukopenie aufgrund einer Lymphopenie

- ggr. Retikulozytose

- ggr. Hyperglykämie

- ggr. Hypercholesterinämie

- ggr. Hypertriglyceridämie

- ggr. Erhöhung von AP und GLDH

- mgr. Erhöhung der ALT

- FeLV/FIV-Schnelltest (SNAP ${ }^{\circledR}$ Kombi Plus FeLV Antigen/FIV Antikörper Test, IDEXX): negativ

Die Ultraschalluntersuchung des Thorax ergab einen geringgradigen Pleuralerguss. Eine Umfangsvermehrung konnte nicht dargestellt werden. Das Abdomen zeigte einen Normalbefund.

In der Tracheoskopie wurde eine ca. $1,5 \times 0,5 \mathrm{~cm}$ große, runde, von dorsal kommende Umfangsvermehrung detektiert ( $\triangleright$ Abb.2), die sich von der Lokalisation mit der Masse in der Röntgenaufnahme des Thorax deckte und das Lumen der Trachea zu 75\% verlegte. Die Oberfläche war glatt, gut vaskularisiert und von gleicher Textur wie die umliegende Schleimhaut.

Computertomografisch zeigte sich keine Beteiligung der umliegenden Strukturen, jedoch war eine Abgrenzung der Masse zum dorsalen Anteil der Trachea nicht nachvollziehbar. Außerdem stellte sich eine weitere, ca. 0,5 $\times 0,3 \mathrm{~cm}$ große, zum Weichteilgewebe isoattenuierte Struktur am Stammbronchus des rechten Mittellappens dar. Es wurde aufgrund der Befunde eine Neoplasie mit Metastasierung in den rechten Mittellappen vermutet.

Zytologisch wurde ein tracheales malignes Lymphom diagnostiziert ( $\triangleright$ Abb.3). In der pathohistologischen Untersuchung mit Typisierung konnte eine Infiltration mit B-Lymphozyten festgestellt werden. Differenzialdiagnostisch kam eine reaktive Hyperplasie oder ein gut differenziertes Lymphom infrage.

\section{Therapie und Verlauf}

Die Katze wurde mit L-Asparaginase behandelt. Eine Röntgenaufnahme am Folgetag zeigte bereits eine partielle Remis-

Tab. 3 CHOP-basiertes Kombinationschemotherapieprotokoll.

\begin{tabular}{|c|c|c|}
\hline Woche & Medikament & Dosis \\
\hline 1 & $\begin{array}{l}\text { Vincristin } \\
\text { L-Asparaginase (optional) } \\
\text { Prednisolon* }\end{array}$ & $\begin{array}{l}0,7 \mathrm{mg} / \mathrm{m}^{2} \text { i. v. } \\
400 \mathrm{IE} / \mathrm{kg} \text { KM s.c. } \\
2 \mathrm{mg} / \mathrm{kg} \text { KM p.o. } 1 \times \text { täglich }\end{array}$ \\
\hline 2 & Cyclophosphamid & 250 mg/m² p.o. \\
\hline 3 & Vincristin & $0,7 \mathrm{mg} / \mathrm{m}^{2} \mathrm{i} . \mathrm{v}$. \\
\hline 4 & Doxorubicin & $30 \mathrm{mg} / \mathrm{m}^{2}$ i. v. \\
\hline 6 & Vincristin & $0,7 \mathrm{mg} / \mathrm{m}^{2} \mathrm{i} . \mathrm{v}$. \\
\hline 7 & Cyclophosphamid & 250 mg/m² p.o. \\
\hline 8 & Vincristin & $0,7 \mathrm{mg} / \mathrm{m}^{2} \mathrm{i} . \mathrm{v}$. \\
\hline 9 & Doxorubicin & 30 mg/m² i.v. \\
\hline 11 & Vincristin & 0,7 mg/m²i.v. \\
\hline 13 & Chlorambucil & 20 mg/m² p.o. \\
\hline 15 & Vincristin & $0,7 \mathrm{mg} / \mathrm{m}^{2} \mathrm{i} . \mathrm{v}$. \\
\hline 17 & Doxorubicin & $30 \mathrm{mg} / \mathrm{m}^{2}$ i. v. \\
\hline 19 & Vincristin & $0,7 \mathrm{mg} / \mathrm{m}^{2} \mathrm{i} . \mathrm{v}$ \\
\hline 21 & Chlorambucil & 20 mg/m² p.o. \\
\hline 23 & Vincristin & $0,7 \mathrm{mg} / \mathrm{m}^{2} \mathrm{i} . \mathrm{v}$ \\
\hline $25^{* *}$ & Doxorubicin & 30 mg/m² i. v. \\
\hline
\end{tabular}

* $1 \times$ täglich in dieser Dosierung über die gesamte Zeit der Chemotherapie.

** Ist der Patient hier in kompletter Remission, kann die Chemotherapie beendet werden.

sion des Tumors. Am Tag 4 wurde mit einem CHOP-basierten Kombinationschemotherapieprotokoll begonnen (CHOP: $\mathrm{C}=$ Cyclophosphamid, $\mathrm{H}=$ Doxorubicin $\rightarrow$ Hydroxydaunorubicin, $\mathrm{O}=$ Vincristin $\rightarrow$ Oncovin, $\mathrm{P}=$ Prednisolon; $>$ Tab.3). Die Katze wurde in die häusliche Pflege übergeben. Der Patient zeigte 1 Woche später nach Belastung eine pumpende Atmung, die sich in Ruhe wieder normalisierte. In einer daraufhin durchgeführten Kontrollröntgenaufnahme des Thorax zeigte sich weiterhin eine Masse in der Trachea. Die Chemotherapie wurde wie geplant mit Cyclophosphamid weitergeführt. Ab diesem Zeitpunkt zeigte die Katze keine Atemnot mehr. In erneuten Röntgenaufnahmen des Thorax (Woche 3, 13 und 19) war kein Tumor mehr nachvollziehbar. Der Patient befand sich in kompletter Remission.

Insgesamt wurde die Chemotherapie sehr gut vertragen. Erbrechen trat selten auf. Einmal war ein stationärer Aufenthalt

\section{konkret}

Vor der Gabe von Doxorubicin sollten eine blutchemische Untersuchung (Nierenwerte) und eine Urinuntersuchung erfolgen, da Doxorubicin bei der Katze nierentoxisch ist.

über 2 Tage mit Infusionstherapie nötig. In der Woche 9, nach der Gabe von Vincristin, wurde eine hochgradige Neutropenie $\left(0,62 \times 10^{9}\right.$; Referenzbereich: $2,5-$ $12,5 \times 10^{9}$ ) festgestellt. Daher wurde zunächst ein Breitbandantibiotikum (Amoxicillin-Clavulansäure) über 1 Woche verabreicht und die Chemotherapie um 1 Woche verschoben. Das Protokoll wurde nach Normalisierung der Blutwerte wie geplant fortgesetzt, jedoch wurde die Dosis von Vincristin aufgrund der Nebenwirkungen (Neutropenie) jeweils um $25 \%$ reduziert. Trotz der Reduktion zeigte die Katze nach der Gabe von Vincristin immer wieder eine gering- bis hochgra- 
dige Neutropenie, sodass letzten Endes die Gabe von Vincristin in der Woche 23 ausgesetzt wurde, da eine weitere Reduktion unterhalb einer therapeutisch wirksamen Dosis gefallen wäre. Nach insgesamt 25 Wochen Chemotherapie wurde das Protokoll wie geplant beendet.

Die Katze befindet sich 908 Tage nach Therapiebeginn klinisch in kompletter Remission. Die Besitzerin berichtet von einer normalen Lebensqualität. Seit der 2. Woche der Chemotherapie zeigt die Katze keine respiratorischen Symptome mehr.

\section{Praxistipp}

Vor jeder Applikation der Chemotherapie sollte eine Hämatologie angefertigt werden. Bei einer Neutrophilenzahl von $<1500$ Zellen/UL sollte zunächst 5-7 Tage abgewartet werden. Sind die Neutrophilen in der Kontrolle $>1500$ Zellen/UL, kann die Chemotherapie fortgesetzt werden.

\section{Fazit}

Die Diagnosestellung eines Lymphoms geht nicht zwingend mit einer schlechten Prognose einher. Um die optimale Therapieform auszuwählen ist es wichtig, zwischen den einzelnen Formen zu unterscheiden. Eine Chemotherapie ist in der Regel das Mittel der Wahl, aber auch eine Bestrahlungstherapie ist manchmal indiziert. Bei einem trachealen Lymphom können beide Therapien zu einem guten Ergebnis führen. Die Gabe von Glukokortikoiden oder gar eine chirurgische Entfernung des Tumors ist nicht anzuraten. Der anschließende Fallbericht zeigt, dass die Chemotherapie sehr gut vertragen wurde und der Patient noch Jahre danach symptomfrei lebt.

Online zu finden unter

http://dx.doi.org/10.1055/s-0035-1550103

\section{Literatur}

1 Beaumont PR. Intratracheal neoplasia in two cats. J Small Anim Pract 1982; 23 (1): 29-35

2 Brown MR, Rogers KS, Mansell KJ et al. Primary intratracheal lymphosarcoma in four cats. J Am Anim Hosp Assoc 2003; 39 (5): 468-472

3 Dugas B, Hoover J, Pechman R. Computed tomography of a cat with primary intratracheal lymphosarcoma before and after systemic chemotherapy. J Am Anim Hosp Assoc 2011; 47 (6): 131-137

4 Jakubiak MJ, Siedlecki CT, Zenger E et al. Laryngeal, laryngotracheal, and tracheal masses in cats: 27 cases (1998-2003). J Am Anim Hosp Assoc 2005; 41 (5): 310-316

5 Ovejero Braun A, Hauser B. Correlation between cytopathology und histopathology of the skin, lymph node and spleen in 500 dogs and cats. Schweiz Arch Tierheilkd 2007; 149 (6): 249-257

6 Schneider PR, Smith CW, Feller DL. Histiocytic lymphosarcoma of the trachea of a cat. J Am Anim Hosp Assoc 1979; 15: 485-487

7 Teske E. Hämatopoetische Tumoren. In: Kessler M, Kleintieronkologie. Stuttgart: Enke; 2012: 493-521

8 Vail DM. Feline Lymphoma and Leukemia. In: Vail DM, Page RL, Withrow SJ, eds. Withrow and MacEwen's Small Animal Clinical Oncology. St. Louis: Elsevier Health Sciences; 2013: 638-653

9 Zimmerman U, Muller F, Pfleghaar S. Zwei Fälle von histogenetisch unterschiedlichen Trachealtumoren bei Katzen. Kleintierprax 1992; 37: 409-412

\section{Nina Müller}

Gabriel Wurtinger

Klinik für Kleintiere - Innere Medizin

Andreas Moritz

Klin. Pathophysiologie \&

Klin. Laboratoriumsdiagnostik

Justus-Liebig-Universität

Frankfurter Str. 126

35392 Gießen

Nina.Mueller@vetmed.uni-giessen.de

\section{Christina Schwens}

sylab.vet

Paracelsusstraße 13

51375 Leverkusen 